\title{
The Ustilago hordei-barley interaction is a versatile system to characterize fungal effectors
}

\author{
Bilal Ökmen ${ }^{1 *}$, Daniela Schwammbach², Guus Bakkeren³, Ulla Neumann ${ }^{4}$, Gunther \\ $\operatorname{Doehlemann}^{1} *$
}

1 Institute for Plant Sciences, University of Cologne, BioCenter, Zuelpicher Str. 47a, 50674 Cologne, Germany

2 Max Planck Institute for Terrestrial Microbiology, Karl von Frisch Str., D-35043 Marburg, Germany

3 Central Microscopy, Max-Planck-Institute for Plant Breeding Research, 50829 Cologne, Germany

4 Agriculture and Agri-Food Canada, Summerland Research and Development Centre, Summerland, BC, VOH 1Z0, Canada

*Correspondence to: bilal.oekmen@uni-koeln.de; g.doehlemann@uni-koeln.de

\begin{abstract}
Obligate biotrophic fungal pathogens, such as Blumeria graminis and Puccinia graminis, are amongst the most devastating plant pathogens, causing dramatic yield losses in many economically important crops worldwide. However, a lack of reliable tools for the efficient genetic transformation has hampered studies into the molecular basis of their virulence/pathogenicity. In this study, we present the $U$. hordei-barley pathosystem as a model to characterize effectors from different plant pathogenic fungi. We have generated $U$. hordei solopathogenic strains, which form infectious filaments without presence of compatible mating partner. Solopathogenic strains are suitable as heterologous expression system for fungal virulence factors. A highly efficient Crispr/Cas9 gene editing system is made available for $U$. hordei. In addition, $U$. hordei infection structures during barley colonization were analyzed by transmission electron microscopy, which shows that $U$. hordei forms intracellular infection structures sharing high similarity to haustoria formed by obligate rust and powdery mildew fungi. Thus, $U$. hordei has high potential as a fungal expression platform for functional studies of heterologous effector proteins in barley.
\end{abstract}

Key words: Ustilago hordei, heterologous gene expression, effectors, haustoria, CRISPR-Cas9 
bioRxiv preprint doi: https://doi.org/10.1101/2020.12.10.419150; this version posted December 11, 2020. The copyright holder for this preprint (which was not certified by peer review) is the author/funder. All rights reserved. No reuse allowed without permission. 


\section{Introduction}

2 Plant pathogens have evolved different types of pathogenic life-styles with their host, ranging

3 from obligate biotrophic, biotrophic, hemibiotrophic and necrotrophic. For successful

4 colonization, each pathogen deploys a distinct set of effectors that target specific host

5 molecules, pathways and structures. Recent genome and transcriptome analyses of a wide

6 range of phytopathogens provide new insights into the effector inventories of pathogens that

7 have different pathogenic life styles [1-5]. It has been found that compared to biotrophic

8 pathogens, necrotrophs and hemibiotrophs have more plant cell wall degrading enzymes,

9 secondary metabolites and toxins in order to kill their host cells during the infection and feed

10 on nutrients released from dead host cells [6-8]. On the other hand, effector catalogues of biotrophs appear to be more specialized, reflecting their ability to efficiently suppress host defenses including regulated cell death, since their survival strictly depends on living host cells [7, 8]. While many effectors from different facultative biotrophs, hemibiotrophs and necrotrophs have been functionally characterized, there is still limited mechanistic insight into effectors of obligate biotrophic filamentous pathogens. A main reason for this gap is the absence of efficient genetic transformation and gene deletion techniques to perform reverse genetics in obligate biotrophs.

Currently, functional characterization of effectors of obligate biotrophic pathogens is performed via different strategies. Effectors from these pathogens can be heterologously expressed in planta and their positive contribution to virulence can be determined via subsequent inoculation of these plants with several pathogens $[9,10]$. However, heterologous expression of some effectors in planta can result in strong pleiotropic defects that compromise symptom evaluations. In another strategy, the type III secretion system (T3SS) of Pseudomonas syringae (for Arabidopsis) and Pseudomonas fluorescens or Pseudomonas atropurpurea (for wheat and barley) is used for functional characterization of several intracellular effectors from obligate biotrophs like rusts and powdery mildews [11-15]. Any growth promotion observed for Pseudomonas sp. transformants, which deliver the desired effectors into the host cell during infection, is interpreted as a positive contribution to virulence [11-15]. However, the T3SS of Pseudomonas sp. also has some drawbacks. For example, fungal effectors that require post-translational modifications for their activity will not be correctly produced by the Pseudomonas sp. system, since prokaryotes lack the molecular machinery necessary for these modifications. In addition, the T3SS system delivers effectors into host plant cells, and hence fungal effectors that play roles in the apoplast or are required for haustorium formation and function during host colonization, might not be 
identified. Furthermore, the function of some effectors from biotrophic pathogens is to avoid or suppress PAMP-triggered immunity (PTI) to promote disease establishment. PAMPs from bacterial and fungal pathogens are different because of their phylogenetic distance, so unless the signaling pathways that lead to PTI are not completely conserved, PTI responses induced by Pseudomonas sp. may not be evaded or suppressed by fungal effectors. In another method, to validate virulence function of obligate biotrophs' effector genes during host colonization, a host-induced gene silencing (HIGS) assay was developed [16-18]. However, requirements of stable transgenic host lines of HIGS constructs make this method very laborious.

The non-obligate biotrophic fungal pathogen Ustilago hordei is a causal agent of covered smut disease on barley and oat plants. U. hordei belongs to the group of Ustilaginales, members of which infect many economically important crops including maize, wheat, barley, oat and sugar cane. Similar to other smut fungi, pathogenic development of $U$. hordei is coupled to sexual development [19]. For successful infection, two haploid sporidia of opposite mating types fuse to form an infectious dikaryotic filament that subsequently differentiate to form an appressorium, a swollen hyphal cell that leads to direct penetration of host epidermal cells. During plant colonization, $U$. hordei proliferates both extra- and intracellularly and forms haustorium-like feeding structures in the host cells [20]. The $U$. hordei reaches and establishes itself in the host meristem and then grows with the plant until floral meristem develops spikelets, which likely gives a cue to the fungus to multiply and sporulate. Massive proliferation and sporulation of the fugus in the barley inflorescence is displayed by mass production of dark brown smut teliospores [21].

Blumeria graminis f. sp. hordei (Bgh) and Puccinia graminis f. sp. tritici (Pgt) are obligate biotrophic pathogens which are the causal agents of powdery mildew and stem rust on barley, respectively $[22,23]$. Unlike $U$. hordei, which can be cultured in vitro, both Bgh and $P g t$ have obligate biotrophic lifestyles and cannot be cultured outside the host. Therefore, the generation of stable fungal transformants is the main bottleneck to study these pathosystems at the molecular level. Despite their phylogenetic distance, Bgh (Ascomycota), Pgt and $U$. hordei (Basidiomycota) share significant similarities: they are barley or wheat pathogens, establish strictly biotrophic interactions with their host in which they form specialized intracellular feeding-structures, the haustoria [20, 22, 23]. These similarities prompted us to establish cell biological, molecular and genetic methods to use the $U$. hordei-barley as a model system for functional characterization of effector candidates from different filamentous phytopathogens. 


\section{Material and Methods}

Plant and fungal materials

To isolate total genomic DNA from axenic culture, Ustilago hordei (4857-4) strain were incubated in YEPS $_{\text {light }}(0.4 \%$ yeast extract, $0.4 \%$ peptone, and $2 \%$ saccharose) liquid medium at $22^{\circ} \mathrm{C}$ with $200 \mathrm{rpm}$ shaking till OD:1.0. To isolate total gDNA from U. hordei-infected

barley plants (at $6 \mathrm{dpi}$ ), the third leaves of the $U$. hordei infected barley plants were collected by cutting $1 \mathrm{~cm}$ below the injection needle sites. Leaf samples were then frozen in liquid nitrogen and ground using a mortar and pestle under constant liquid nitrogen. The gDNA was isolated by using a MasterPure ${ }^{\mathrm{TM}}$ Complete DNA\&RNA Purification Kit (Epicentre ${ }^{\circledR}$, Illumina $^{\circledR}$, Madison, Wisconsin, USA) according to the manufacturer's instructions.

Susceptible Golden Promise barley cultivar was grown in a greenhouse at $70 \%$ relative humidity, at $22^{\circ} \mathrm{C}$ during the day and the night; with a light/dark regime of $15 / 9 \mathrm{hrs}$ and 100 Watt $\mathrm{m}^{-2}$ supplemental light when the sunlight influx intensity was less than $150 \mathrm{Watt}^{-2}$.

\section{Nucleic acids methods}

Fungal biomass quantification was performed by using quantitative PCR (qPCR) analysis as in Ökmen et al., 2018 [20]. Genomic DNA from infected barley leaves at 6 days postinoculation (dpi) was isolated by using the MasterPure ${ }^{\mathrm{TM}}$ Complete DNA\&RNA purification Kit (Epicentre ${ }^{\circledR}$, Illumina $\left.{ }^{\circledR}\right)$ according to manufacturer's instructions. The U. hordei UhPpi gene (UHOR_05685) was used as a reference gene. A standard curve was constructed by using serial dilutions of $U$. hordei genomic DNA $\left(100,10,1,0.1,0.01,0.001 \mathrm{ng}^{-1} \mathrm{l}^{-1}\right)$ using UhPpi as a reference gene. Base 10 logarithms of DNA concentrations were plotted against the crossing point of $\mathrm{Ct}$ values. The $\mathrm{qPCR}$ reaction was performed in a Bio-Rad iCycler system by using the following program: $2 \mathrm{~min}$ at $95^{\circ} \mathrm{C}$ followed by 45 cycles of $30 \mathrm{~s}$ at $95^{\circ} \mathrm{C}$, $30 \mathrm{~s}$ at $61^{\circ} \mathrm{C}$ and $30 \mathrm{~s} 72^{\circ} \mathrm{C}$. The primers that have been used for qPCR are listed in Table S1. All PCRs reactions were performed by using Phusion (C DNA polymerase (Thermo Scientific; Bonn, Germany) following the manufacturer's instructions with 100 ng genomic DNA or cDNA as template. All primers that are used in PCR reaction for cloning of different genes are listed in Table S1. The amplified DNA fragments were then used for cloning processes. All PCR reaction took place in a PTC-200 (Peletier Thermal Cycler, MJ Research) PCR machine. Nucleic acids deriving from PCRs or restriction digests reactions were purified from 1\% TAE agarose gels with the "Wizard SV Gel and Purification System Kit" (Promega) 
103

104

105

106

107

108

109

110

111

112

113

114

115

116

117

118

119

120

121

122

123

124

125

126

127

128

129

130

131

132

133

134

135

136

according to the manufacturer's instructions. Plasmid isolation from bacterial cells was performed by using the QIAprep Mini Plasmid Prep Kit according to the manufacturer's information.

\section{Construction of expression vectors}

For heterologous gene expression constructs (p123-pUHOR02700::SP-Gus-mCherry, p123pUHOR02700::Gus-mCherry, p123-pUHOR02700::SP-FvRibol and p123-pActin::SPCfAvr4), standard molecular biology methods were used according to molecular cloning laboratory manual of Sambrook et al. (1989) [24]. Amplified PCR fragments for each gene (Gus-mCherry, FvRibol and CfAvr4) were cut with appropriate restriction enzymes, subsequently they were ligated into a vector that was digested with the same restriction enzymes by using T4-DNA ligase (New England Biolabs; Frankfurt a.M., Germany) according to manufacturer's instructions. The sequences confirmation of each construct was performed via sequencing at the Eurofins Genomics (Cologne, Germany). All vector constructs, primer pairs and restriction sites are indicated in Table S1. Escherichia coli transformation was performed via heat shock assay according to standard molecular biology methods [24].

\section{CRISPR/Cas9 gene editing system}

To establish the CRISPR/Cas9-HF (high fidelity) gene editing system in the $U$. hordei, a plasmid containing codon optimized Cas $9-H F$ gene under the control of $H s p 70$ promoter and carboxin resistance was used from Zuo et al. (2020) [25]. To express sgRNA for targeted gene, Ustilago maydis pU6 promotor was replaced with the U. hordei $p U 6$ promotor. The sgRNAs for the knockout of the $U$. hordei gene was designed by E-CRISPR (http://www.ecrisp.org/ECRISP/ aboutpage.html) (Table S1) [26]. Plasmid construction for CRISPR/Cas9 was performed as described by Zou et al. (2020). The CRISPR/Cas9-HF vector was linearized with restriction enzyme Acc65I, and subsequently assembled with spacer oligo and scaffold RNA fragment with 3' downstream 20 bp overlap to the plasmid by using Gibson Assembly [27].

\section{Fungal transformation and virulence assays}

The U. hordei transformation assay was conducted by using protoplasts according to Kämper, (2004) [28]. Virulence assays for DS200-FvRibo1 and DS200 U. hordei strains were performed according to Ökmen et al., 2018. Briefly, all U. hordei strains were grown in 
137 YEPS $_{\text {light }}$ liquid medium at $22^{\circ} \mathrm{C}$ and $200 \mathrm{rpm}$ shaking until getting an $\mathrm{OD}_{600}$ of $0.6-0.8$.

138 Subsequently, U. hordei cells were centrifuged at $3500 \mathrm{rpm}$ for $10 \mathrm{~min}$ at RT and resuspended in sterile distilled water supplemented with $0.1 \%$ Tween-20 to an $\mathrm{OD}_{600}$ of 3.0. Then each $U$. hordei cell suspension was injected into stems of 12-day-old barley seedlings (Golden Promise) with a syringe with needle. All infection assays were performed in three biological replicates with at least 15 plants. Fungal biomass quantification of $U$. horde $i$ was performed at 6 dpi by using genomic DNA (200 ng $\mu \mathrm{l}^{-1}$ ) as the template with qPCR. To confirm secretion of GUS-mCherry protein in apoplast of barley leaf, apoplastic fluid (AF) from $U$. hordei DS200 \pm GUS-mCherry strains infected barley leaves were isolated according to van der Linde et al. (2012) [29]. After AF isolation, western blot analysis was performed to detect GUS-mCherry signal in isolated AFs. Western blot was performed as described in Mueller et al. (2013) [30]. To confirm that $U$. hordei can express and secrete functional effector proteins from different fungi, Avr4 of Cladosporium fulvum and Ribol (encoding a secreted ribotoxin) of Fusarium verticillioides were heterologously expressed in the $U$. hordei strain DS200 with the UHOR_O2700 signal peptide (SP) and under control of pActin (for in vitro expression) and pUHOR_02700 (for in planta expression) promoters, respectively. To confirm expression and secretion of CfAvr4 effector protein in $U$. hordei in vitro, culture filtrates isolated from DS200-CfAvr4, DS200-FvRibo1 and DS200 strains (OD:1.0) were collected with centrifugation (13000 rpm for $5 \mathrm{~min}$ ). After filter sterilization, each culture filtrate was infiltrated in tobacco leaves expressing $C f 4$ resistance gene (a gene encoding tomato Cf4 receptor protein that can recognize CfAvr4 protein and induce hypersensitive response) to induce hypersensitive response.

\section{Light Microscopy}

161 The wheat germ agglutinin (WGA)-AF488 (Molecular Probes, Karlsruhe, Germany) and 162 propidium iodide (PI) (Sigma-Aldrich) staining was performed according to Ökmen et al., 1632018 [20]; WGA-AF488 stains fungal cell walls and the propidium iodide stains plant cell 164 walls. $U$. hordei infected barley leaves were first bleached in pure ethanol and then they were boiled for 1-2 hours in $10 \% \mathrm{KOH}$ at $85^{\circ} \mathrm{C}$. Subsequently, the $\mathrm{pH}$ of boiled leaf samples was neutralized by using 1xPBS buffer ( $\mathrm{pH}$ : 7.4) with several washing steps. Then, the WGAAF488/PI staining solution $\left(1 \mu \mathrm{g} \mathrm{ml}^{-1}\right.$ propidium iodide, $10 \mu \mathrm{g} \mathrm{ml}^{-1}$ WGA-AF488; $0.02 \%$ Tween 20 in PBS pH 7.4) was vacuum infiltrated in samples for 5 min at 250 mbar using a desiccator (vacuum infiltration step was performed three times). The WGA-AF488/PI stained leaf samples were stored in $1 x P B S$ buffer $(\mathrm{pH}: 7.4)$ at $4^{\circ} \mathrm{C}$ in the dark until microscopy. 
171 WGA-AF488: excitation at $488 \mathrm{~nm}$ and detection at 500-540 nm. PI: excitation at $561 \mathrm{~nm}$ and 172 detection at $580-630 \mathrm{~nm}$.

173 To visualize secretion of GUS-mCherry protein in $U$. hordei during barley colonization, $U$. 174 hordei DS200 \pm GUS-mCherry strains were inoculated on barley plants. Subsequently, 175 infected barley leaves were checked for localization of GUS-mCherry at 4 dpi by using a 176 Leica SP8 confocal microscopy. For mCherry fluorescence of hyphae in barley tissue, an 177 excitation at $561 \mathrm{~nm}$ and detection at 580-630 $\mathrm{nm}$ was used.

\section{Transmission electron microscopy}

180 Chemically fixed samples were prepared according to Wawra et al., (2019) with minor changes [31]. For TEM observation, $2 \mathrm{~mm}$ leaf discs from infected and non-infected Hordeum vulgare leaves were excised from $1 \mathrm{~cm}$ below infection sites by using a biopsy punch and chemically fixed in $2.5 \%$ glutaraldehyde and $2 \%$ paraformaldehyde in $0.05 \mathrm{M}$ sodium cacodylate buffer, $\mathrm{pH} 6.9$, supplemented with $0.025 \% \mathrm{CaCl} 2(\mathrm{w} / \mathrm{v})$ for $2 \mathrm{~h}$ at room temperature. Subsequently, samples were rinsed six times for 10 minutes in $0.05 \mathrm{M}$ sodium cacodylate buffer ( $\mathrm{pH} 6.9$, rinse 3 and 4 supplemented with $0.05 \mathrm{M}$ glycine) and post-fixed for $1 \mathrm{~h}$ at room temperature with $0.5 \% \mathrm{OsO} 4$ in $0.05 \mathrm{M}$ sodium cacodylate buffer, $\mathrm{pH} 6.9$, supplemented with $0.15 \%$ potassium ferricyanide. After thorough rinsing in $0.05 \mathrm{M}$ sodium cacodylate buffer ( $\mathrm{pH}$ 6.9) and water, samples were dehydrated in an ethanol series from 10\% to $100 \%$, gradually transferred to acetone and embedded into Araldite 502/Embed 812 resin (EMS, catalog number 13940) using the ultra-rapid infiltration by centrifugation method revisited by McDonald (2014) [32].

For TEM observation, leaf samples were also processed by means of high pressure freezing and freeze substitution as an alternative to conventional chemical fixation following the procedure described in Micali et al. (2011) for ultrastructural observations [33]. Once the samples reached room temperature, they were rinsed in acetone, carefully removed from the aluminium specimen carriers and gradually infiltrated in LR White resin (Plano $\mathrm{GmbH}$ ) for 6 days. Resin polymerization was done in flat embedding molds at $100^{\circ} \mathrm{C}$ for $24 \mathrm{~h}$. Ultrathin (70-90 nm) sections were collected on nickel slot grids as described by Moran and Rowley (1987) [34], stained with $0.1 \%$ potassium permanganate in $0.1 \mathrm{~N} \mathrm{H} 2 \mathrm{SO} 4$ [35], followed by $2 \%(\mathrm{w} / \mathrm{v})$ aqueous uranyl acetate and lead citrate for $15 \mathrm{~min}$ [36] and examined with an Hitachi H-7650 TEM (Hitachi High-Technologies Europe GmbH, Krefeld, Germany) 
bioRxiv preprint doi: https://doi.org/10.1101/2020.12.10.419150; this version posted December 11, 2020. The copyright holder for this preprint (which was not certified by peer review) is the author/funder. All rights reserved. No reuse allowed without permission.

204 Techniques, Danvers, USA). Immunogold labelling of B-1,3-glucan was done according to 205 the procedures described previously [33].

206

207 
208

209

210

211

212

213

214

215

216

217

218

219

220

221

222

223

224

225

226

227

228

229

230

231

232

233

234

235

236

237

238

239

240

241

\section{Results}

\section{Construction of solopathogenic strain of Ustilago hordei}

The requirement of mating for the induction of pathogenic development implies that genetic mutations always need to be made in two compatible $U$. hordei strains, which presents an obvious drawback of the system, particularly for larger scale analyses. To optimize the work flow, we have generated a solopathogenic $U$. hordei strain, which does not require a mating partner to form an infectious filament (Figure 1A-D). For pathogenic development, $U$. hordei requires both a compatible pheromone (Mfa)/pheromone receptor (Pra) pair and an active heterodimer made from $b E$ and $b W$ gene products [37, 38]. To construct solopathogenic $U$. hordei strains, the $b E 1$ allele from mating type locus $1(M A T-1)$ were replaced with the $b E 2$ allele from mating type locus 2 (MAT-2), or both $M f a 1$ and $b E 1$ alleles from mating type locus 1 (MAT-1) were replaced with $M f a 2$ and $b E 2$ alleles from mating type locus 2 (MAT-2). While the constructed DS199 solopathogenic strain had a compatible $b$-locus with $b E 2$ and $b W 1$ genes from different mating types, the DS200 solopathogenic strain contained both compatible MFA2/PRA1 and bE2/bW1 pairs to facilitate the formation of infectious filaments in the absence of a mating partner. For generation of DS199 and DS200 strains, homologous recombination constructs with an FRT-flanked hygromycin resistance cassette (for $M f a 2$ construct) or phleomycin resistance cassette (for $b E 2$ construct), were used, which were removed from the genome after induction of the FRT recombinase [39].

Both the DS199 and DS200 solopathogenic strains were then used for further analysis. To show filamentation ability of solopathogenic strains, single $U$. hordei 4857-4 MAT-1 and 4857-5 MAT-2 strains, mixed 4857-4 MAT-1X4857-5 MAT-2, and solopathogenic strains were grown on PD agar plates containing charcoal. While single 4857-4 MAT-1 and 4857-5 MAT-2A strains were not filamentous, mated 4857-4 MAT-1X4857-5 MAT-2 and solopathogenic strains were fully filamentous on PDA charcoal plates (Figure 1A). To test whether the solopathogenic strains form infection structures (hyphal tip swellings; appressoria) that are required for host penetration, mated wild-type U. hordei 4857-4 MAT-1 and 4857-5 MAT-2, and solopathogenic strains were sprayed on parafilm, which previously has been shown to artificially induce appressorium formation in Ustilago maydis [40]. This showed that both solopathogenic strains form appressoria that are comparable to wild-type strain (Figure 1B). Quantification of appressoria formation on barley leaves revealed that there is no significant difference in appressoria formation (Figure 1D) and penetration efficiency (Figure 1E) of wild-type and solopathogenic strains. Wheat germ agglutininAF488/propidium iodide (WGA-AF488/PI) staining of wild-type and solopathogenic strains 
242 infecting barley leaves revealed that there is no visible difference in colonization at 3 days 243 post-inoculation (dpi), i.e. all three strains were found to colonize the host mesophyll tissue

244 (Figure 1C). While the leaf colonization assay did not show obvious differences between 245 solopathogenic and wild-type strains, in barley seed infection assays, the solopathogenic 246 strain was rarely found to have colonized the barley inflorescence and produce teliospores 247 (after 3-4 months post inoculation) (Figure S1).
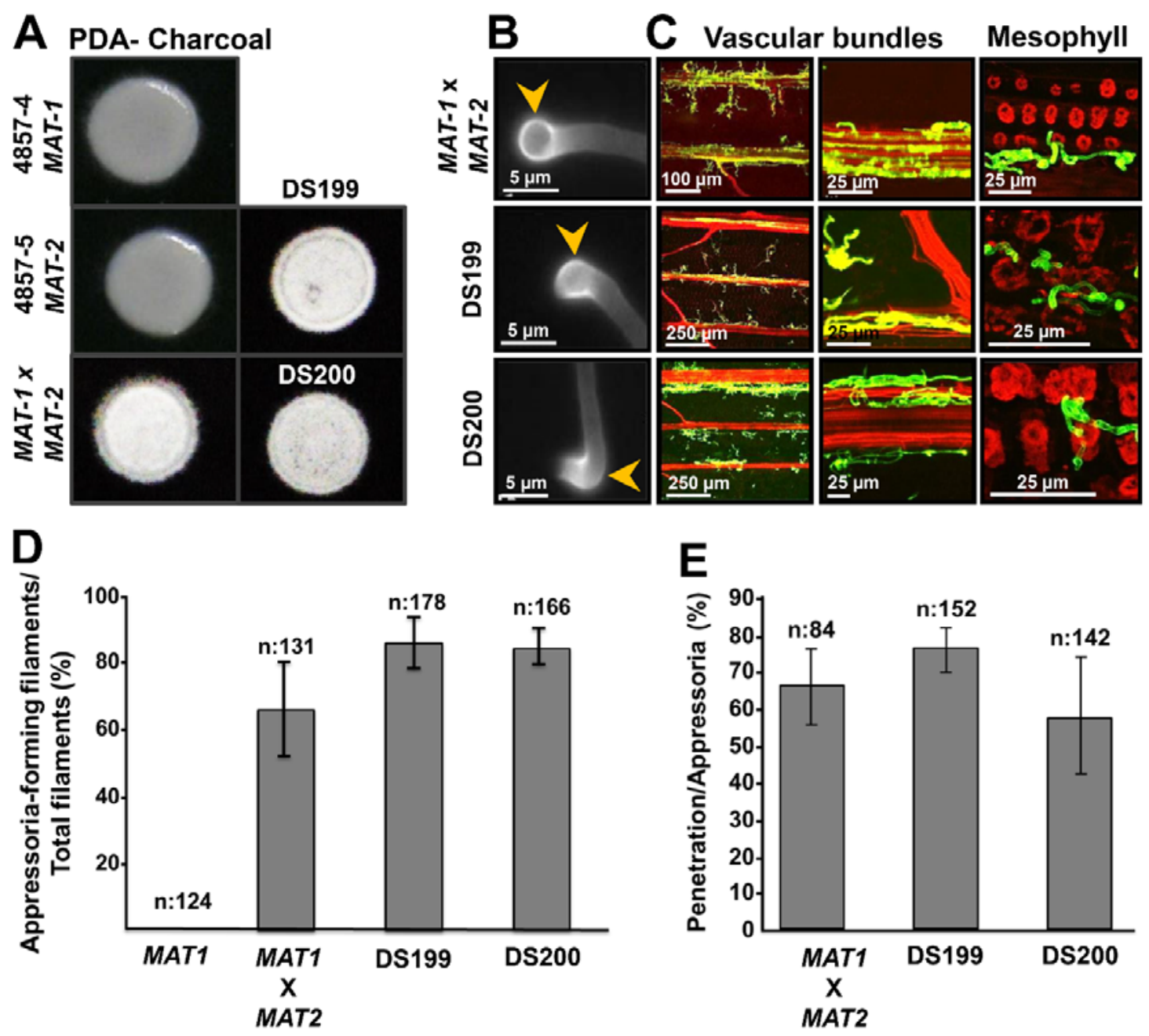

Figure 1 Generation of a solopathogenic Ustilago hordei strain (A) Filamentation test on charcoal plate. $U$. hordei wild-type strains 4857-4 MAT-1, 4857-5 MAT-2, mating of 4857-4 MAT-1X4857-5 MAT-2, solopathogenic DS199 and DS200 strains. Pictures were taken after 3 days incubation at RT. (B) Appressoria formation ability of Ustilago hordei strains on parafilm. Mating of $U$. hordei wild-type 4857-4 MAT-1 and 4857-5 MAT-2, solopathogenic DS199, solopathogenic DS200. Yellow arrowheads indicate appressoria. Pictures were taken after 24 hours incubation (C) Disease development of different Ustilago hordei strains on barley. Mating of $U$. hordei wild-type 4857-4 MAT-1 and 4857-5 MAT-2 at 3 dpi, solopathogenic DS199 at 3 dpi, solopathogenic DS200 at 3 days post inoculation (dpi). Following WGA-AF488/Propidium iodide staining, fungal cell walls are shown in green and plant cell walls in red. (D) Quantification of appressoria formation for $\boldsymbol{U}$. hordei wild-type 4857-4 MAT1, solopathogenic DS199 and DS200 on plant. (E) Quantification of penetration efficiency for $\boldsymbol{U}$. hordei wild-type 4857-4 MAT1, solopathogenic DS199 and DS200 on plant. 
Ultrastructure of the Ustilago hordei-barley interphase during biotrophic interaction

Similar to powdery mildews and rusts, smut fungi including $U$. hordei are considered to be intracellular pathogens. However, in all these interactions, the host plasma membrane is not breached and thus the host-pathogen interaction takes place through the biotrophic interphase, which consists of fungal cell wall (FCW), extracellular matrix (ECM) and plant cell wall (in some regions) (Figure 2A-B). Both chemically fixed as well as high pressure-frozen samples were used to perform transmission electron microscopy (TEM) to display ultrastructural features of the $U$. hordei-barley biotrophic interphase during infection. Samples were taken at 8 dpi, when $U$. hordei growth is primarily intracellular and hyphae can be found in epidermal cells, mesophyll cells and in vascular bundles. Transmission electron micrographs show fungal hyphae growing inside plant cells and frequently branching (Figure S2A-D). The fungal hyphae contain free ribosomes, strands of endoplasmic reticulum, mitochondria, nuclei, as well as lipid bodies, vesicles and vacuoles (Figure S3A-F). Transmission electron micrographs also show the presence of closely paired nuclei in fugal hyphae, which are intimately associated with mitochondria (Figure S3E). Vesicles and multivesicular bodies were also frequently observed at hyphal tips and in the plant cytoplasm adjacent to fungal penetration sites, respectively (Figure 2C-D and Figure S3B, C, F).

It caught our attention that the $U$. hordei hyphal cell wall appears to be surrounded by a two layered extracellular matrix of different electron density: an inner electron-dense (edECM) and an outer electron-translucent layer (etECM), both of unknown composition (Figure 2AB, Figure S3A-C). Immunogold gold labeling with a monoclonal antibody specific for (1-3)particularly prominent (between 50-500 nm thickness) when the hyphae were in contact with the plant cell wall (Figure 2H, yellow arrowheads, Figure S2A, D). In some areas, the middle lamella of the plant cell wall close to the interface was more electron-dense than in areas where it was not in contact with the fungal hypha (Figure $\mathbf{2 H}$, red arrowhead). Another interesting observation of our TEM analysis was that at the site of cell-to-cell penetration, the $U$. hordei hypha is swollen, which resembles appressorial structures (Figure 2G and Figure S2C). During barley colonization, $U$. hordei also forms structures similar to the haustoria known for obligate biotrophs, where they are described to function as feeding structures (Figure 2I-M). Haustorial structures of $U$. hordei were distinguished from the normal hyphae by their bigger size and inter-connected lobular shapes (Figure 2I-L). High magnification transmission electron micrographs of $U$. hordei haustoria showed that these 

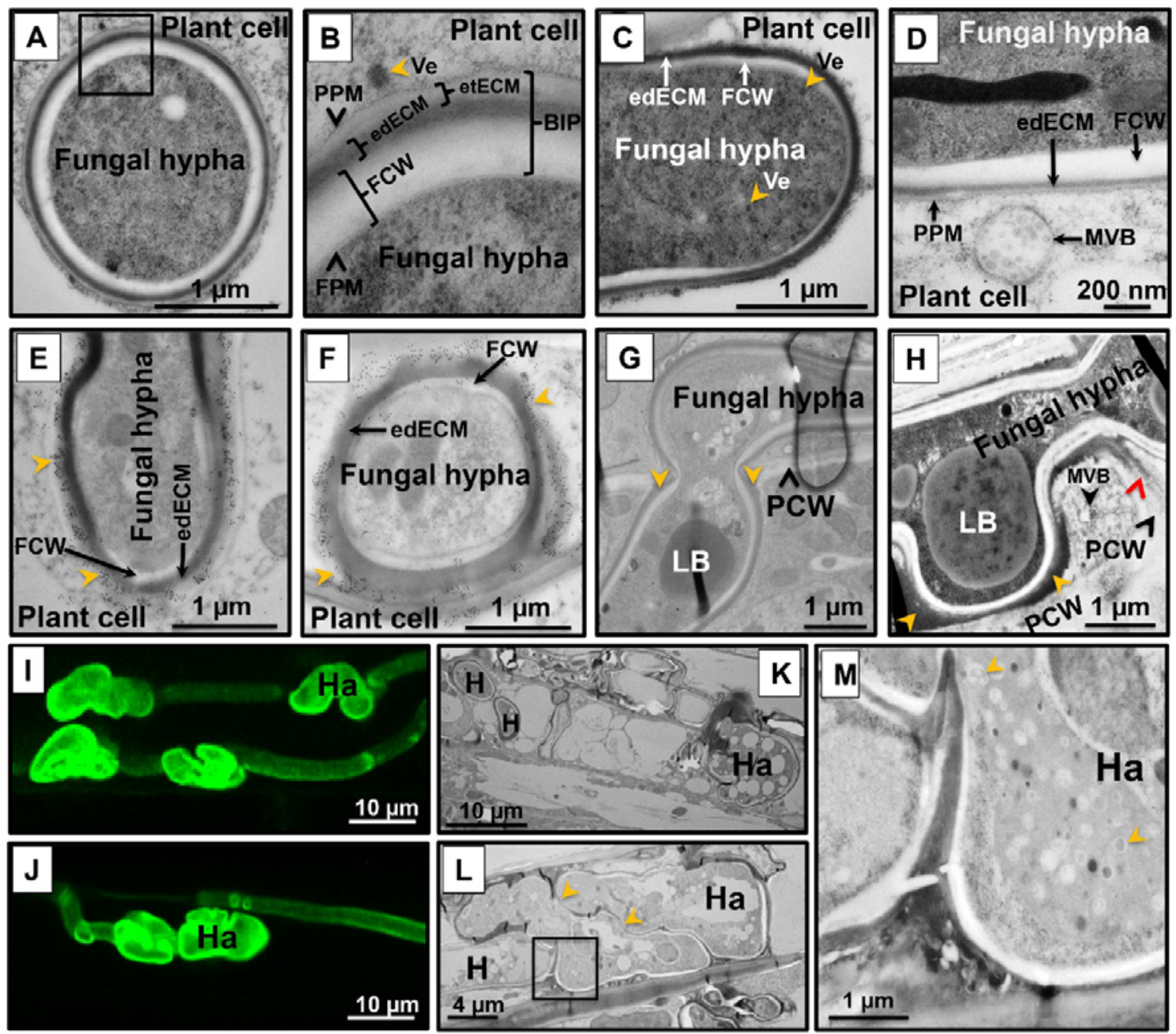

Figure 2 (A-H) Transmission electron microscopy micrographs of wild-type Ustilago hordei infected barley leaves. (A, B) Biotrophic interphase in the $U$. hordei-barley interaction. During host colonization, $U$. hordei invaginates the host cell membrane without breaching it. The host-pathogen interaction mainly takes place within this biotrophic interphase (BIP), which consists of fungal cell wall (FCW), electron-dense extracellular matrix (edECM) and electron-translucent extracellular matrix (etECM). (C, D) Formation of vesicles at the hyphal tip of $\boldsymbol{U}$. hordei. Fungal vesicles (Ve) with cores of different electron density and plant multivesicular bodies (MVB) were detected at hyphal tips and in the plant cytoplasm close to fungal penetration sites, respectively. (E, F) Immunogold labeling of callose with a monoclonal antibody recognizing (1-3)- $\beta$ glucan epitopes. Callose accumulation was detected at the electron-translucent ECM (IdECM) site (yellow arrowheads). (G, H) Cell-to-cell penetration of $\boldsymbol{U}$. hordei. $U$. hordei primarily grows intracellularly at 8 dpi in barley leaves. When the fungal hyphae penetrate a new plant cell, the hypha gets thickened at the site of cell-tocell passage, resembling appressorial structures (G). The edECM gets thicker where the hypha was in contact with the plant cell wall (yellow arrowheads) $(\mathbf{H})$ and electron-dense material can also diffuse into adjacent part of the plant cell wall (red arrowhead) (H). (I-M) Haustoria formation during host colonization. U. hordei grows intracellularly and forms haustorial structures in barley cells. (I, J) WGA-AF488/Propidium iodide staining was performed to visualize $U$. hordei at 8 dpi under confocal/ fluorescent microscopy. (K-M) Transmission electron micrographs showing different planes of section through haustoria. Haustorial structures were distinguished from hyphae by their bigger size and inter-connected lobular shapes. Yellow arrowheads (L) point out the connections between haustorial lobes. U. hordei haustoria possess large vacuoles with a granular lumen containing vesicles of different size (M, yellow arrowheads; magnification of inset in L). BIP: Biotrophic Interphase; FCW: Fungal Cell Wall; FPM: Fungal Plasma Membrane; H: Hypha; Ha: 
Haustorium; edECM: electron-dense extracellular matrix; etECM: electron-translucent extracellular matrix; LB: Lipid bodies; MVB: Multi Vesicular Body; PCW: Plant Cell Wall; PPM: Plant Plasma Membrane; Ve: Vesicles.

\section{Heterologous gene expression in Ustilago hordei}

To use the $U$. hordei-barley pathosystem for functional characterization of secreted virulence factors, heterologous gene expression was established in this smut fungus. As a proof of concept, mCherry fused to the Escherichia coli GusA gene under the control of the U. hordei UHOR_02700 promoter (highly induced upon barley penetration) was heterologously expressed in the ip $(c b x)$ locus of the solopathogenic DS200 strain, either with (+) or without (-) signal peptide (from UHOR_02700) for extracellular secretion (Figure 3A). Confocal microscopy imaging was performed with DS200 strains expressing \pm SP-GusA-mCherry on barley leaves at 3 dpi to monitor expression and localization of recombinant proteins. While SP-GusA-mCherry was localized around the hyphal tip region (showing secretion from the biotrophic hypha), -sp-GusA-mCherry was localized inside the fungal cytoplasm (Figure 3A). Furthermore, western blot analysis was performed with apoplastic fluid isolated from barley leaves infected with \pm SP-GusA-mCherry DS200 strains to confirm secretion of the recombinant proteins. Western blot results also showed that while the secreted full-length SPGusA-mCherry $(\sim 100 \mathrm{kDa})$ and cleaved free mCherry $(\sim 27 \mathrm{kDa})$ were detected in isolated apoplastic fluid, the cytoplasmic -sp-GusA-mCherry was not detectable in isolated apoplastic fluid (Figure 3B).
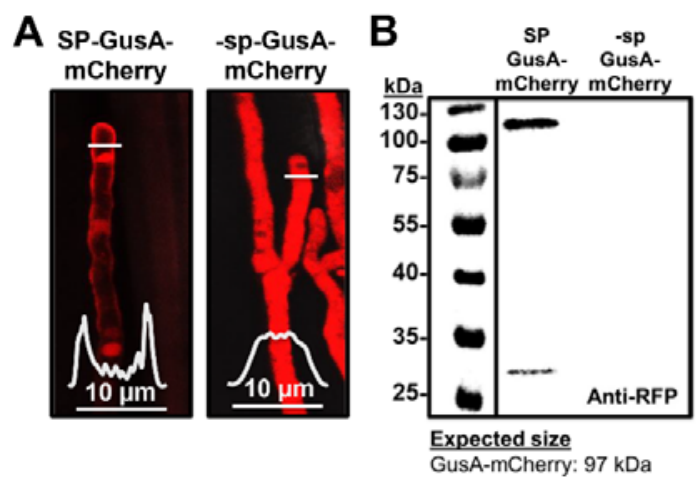

C CRISPR/Cas9 gene editing in $U$. hordei

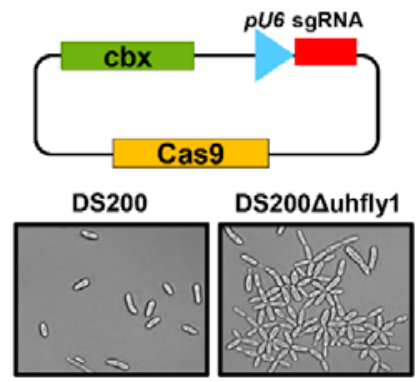

Fig 3. (A-B) Heterologous expression of GusA-mCherry in Ustilago hordei. (A) GusA-mCherry was heterologously expressed in solopathogenic strain DS200 under control of the UHOR_02700 promotor with or without signal peptide for extracellular secretion. \pm SP-GusA-mCherry DS200 strains were inoculated on barley seedlings and at 4 dpi confocal microscopy was performed to monitor expression and localization of recombinant proteins. While +SP-GusA-mCherry is secreted around the tip of the invasive hyphae, -sp-GusAmCherry localizes in the fungal cytoplasm. The white graphs indicate the mCherry signal intensity along the diameter of the hyphae (illustrated by white lines in the image). (B) Western blot analysis was performed with apoplastic fluid isolated from barley leaves infected with \pm SP-GusA-mCherry DS200 strains. While a band corresponding to secreted +SP-GusA-mCherry (at $\sim 100 \mathrm{kDa}$ ) and free mCherry (at $27 \mathrm{kDa}$ ) in isolated apoplastic fluid was detected, no band corresponding to cytoplasmic -sp-GusA-mCherry could be detected. AntiRFP antibody was used for western blot. (C-D) Establishment of CRISPR/Cas9 gene editing system for Ustilago hordei. (C) Codon optimized Cas 9 was cloned into p123 plasmid under the control of Hsp70 promoter. 
The $U$. hordei $p U 6$ promotor was used to express sgRNA for the targeted gene. Carboxin resistance was used as selection marker. (D) U. hordei Flyl gene, a fungalysin metalloprotease involved in fungal cell separation, was edited via CRISPR/Cas9 system for knock-out. While DS200 sporidia showed normal growth, DS200Auhfly1 cells were impaired in cell separation.

\section{CRISPR/Cas9 gene editing in Ustilago hordei}

To establish the CRISPR/Cas9-HF (high fidelity) gene editing system in U. hordei, a codon optimized Cas9-HF gene under the control of $H s p 70$ promoter was expressed in the solopathogenic strain DS200. The U. hordei pU6 promotor was used to express sgRNA of a targeted gene (Figure 3C). As a proof of concept, the U. hordei Flyl gene, a fungalysin metalloprotease involved in fungal cell separation in U. maydis [41], was edited via CRISPR/Cas9 system to result in a truncated protein (after aa 25, a stop codon was introduced). After sequence confirmation of the mutants, microscopic observations showed that while the DS200 strain formed normal yeast cells, DS2004fly1 strains were impaired in cell separation in liquid medium indicating functional conservation of Fly1 in both $U$. hordei and U. maydis (Figure 3D). 83.3 ( \pm 8.3$) \%$ selected independent transformed colonies showed an impaired cell separation phenotype, indicating high efficiency of this method in $U$. hordei.

\section{Activity of heterologous virulence factors expressed in $\boldsymbol{U}$. hordei}

To show that $U$. hordei can express and secrete functional proteins from different plant pathogenic fungi, Avr4 from Cladosporium fulvum (a chitin-binding Avr protein that can be recognized by tomato resistance protein Cf4) and Ribol (encoding a secreted ribotoxin) from Fusarium verticillioides, were heterologously expressed in the DS200 strain. For secretion from $U$. hordei hyphae, the open reading frames were fused with the sequence encoding UHOR_02700 signal peptide (SP). For constitutive expression, heterologous genes were expressed under control of the pActin promoter, and for specific transcriptional induction during plant colonization, the promoter $p U H O R \_02700$ (highly expressed in planta U. hordei effector gene) was used [20]. To confirm in vitro expression and secretion of CfAvr4 effector protein in $U$. hordei, culture filtrates isolated from DS200-CfAvr4, DS200-FvRibo1 and DS200 strains were collected and infiltrated in Nicotiana benthamiana leaves expressing the Cf4 resistance gene, a gene encoding the tomato $\mathrm{Cf} 4$ receptor protein which recognizes the CfAvr4 protein and induce hypersensitive response [42]. While neither DS200 nor DS200FvRibo1 (expressed only in planta) culture filtrates did induce any hypersensitive responsemediated cell death in Cf4 expressing tobacco leaves, the culture filtrate of DS200-CfAvr4 induced hypersensitive response-mediated cell death in the presence Cf4 (Figure 4A). Since Avr4-triggered HR cannot be observed in barley, we deployed FvRibo1 to test secretion of a functional heterologous virulence factor in planta. Heterologous expression of plant cytotoxic 
391 FvRibo1 protein in the DS200 strain was expected to negatively affect the growth of $U$.

392 hordei on barley leaves. Macroscopic observations of infected barley leaves at 6 dpi showed 393 that infection by DS200 strain causes spreading chlorosis along the leaf veins, reflecting 394 spread of fungal proliferation (Fig 4C). In contrast, DS200-FvRibo1 infected barley leaves 395 displayed accumulated focal necrotic spots, reflecting restriction of fungal proliferation 396 (Figure 4C). WGA/PI staining of infected barley leaves revealed that DS200-FvRibo1 strain 397 is mostly restricted to the penetration area and rarely reaches the vascular bundles, while 398 DS200 colonized leaf veins and successfully accessed the host vascular bundles at 6 dpi 399 (Figure 4D). In line with this, fungal biomass quantification of the DS200-FvRibo1 and 400 DS200 strains on infected barley leaves at 6 dpi confirmed a significant virulence reduction of 401 the DS200-FvRibo1 compared to DS200 strain (Figure 4B). Together, these findings show 402 that heterologous expression of FvRibol attenuated $U$. hordei infection (Figure 4B-D).

403
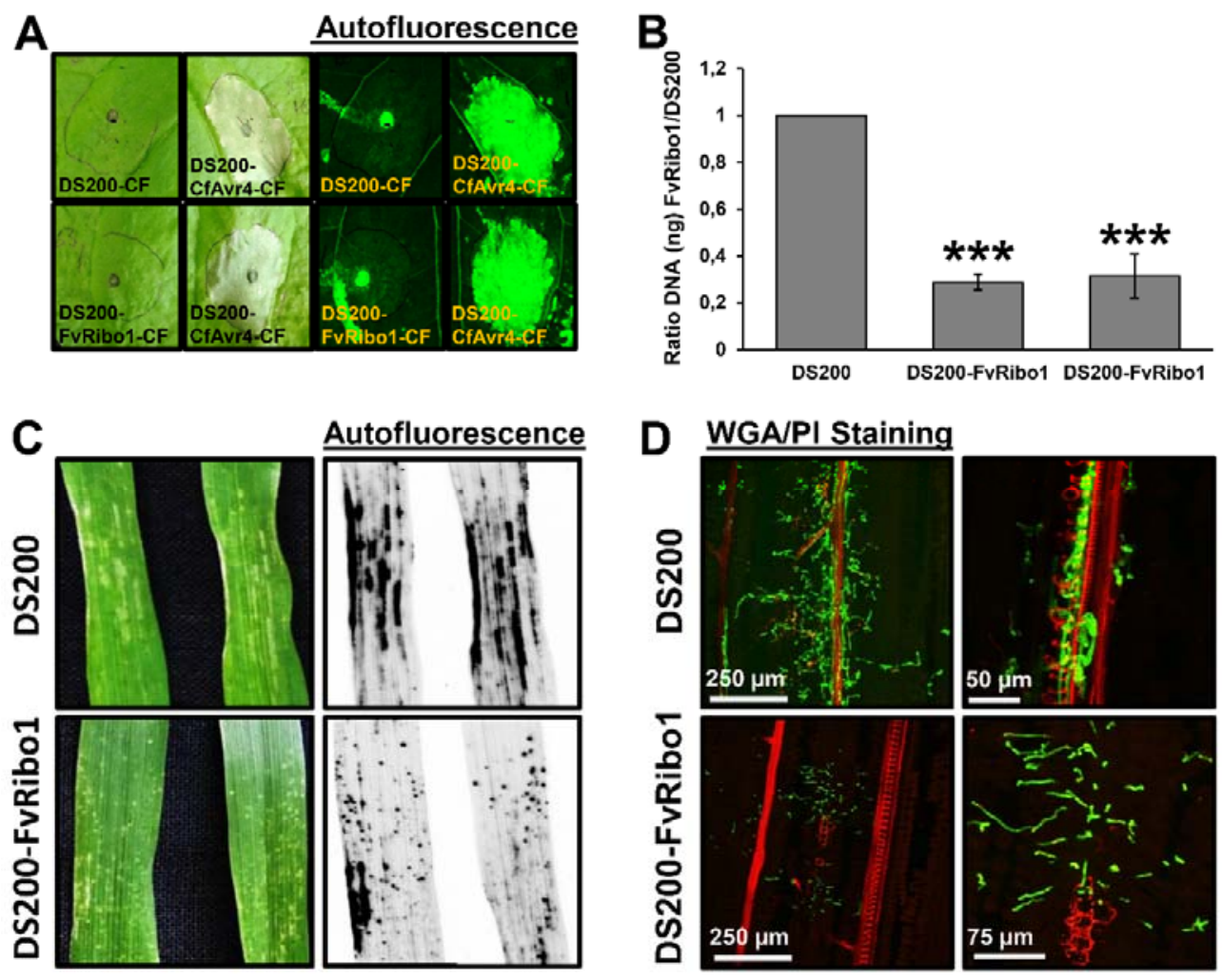

Figure 4 Heterologous expression of fungal effectors in Ustilago hordei. (A) Heterologous expression and secretion of CfAvr4 in $\boldsymbol{U}$. hordei DS200 strain in vitro. U. hordei strain DS200 expressing CfAvr4 of Cladosporium fulvum with UHOR_O2700 signal peptide and under the control of pActin promoter (for constitutive expression), and FvRibo1 of Fusarium verticillioides with UHOR_02700 signal peptide and under the control of $p U H O R \_02700$ promoter (for expression in planta only) were grown in YEPS light $_{\text {liquid medium }}$ till OD:1.0. The $U$. hordei cell suspensions were centrifuged and the culture filtrates (CF) of each sample were infiltrated into tobacco leaves expressing Cf4 resistant protein that can recognize CfAvr4 and induce cell death by means of hypersensitive response. The culture filtrates from $U$. hordei DS200 and DS200-FvRibo1 strains were used as negative controls. Pictures were taken at $5 \mathrm{dpi}$. Autofluorescence of infected leaves was imaged to 
more easily see sites of cell death by using Gel-Doc (Bio-Rad). (B) Biomass quantification of DS200-FvRibo1 in barley leaves. The virulence of the $U$. hordei DS200 and two independent DS200-FvRibo1 strains was assessed by fungal biomass quantification from DNA isolated from infected barley leaves at 6 dpi. The Ppil gene of $U$. hordei was used as a standard for qPCR. The fungal biomass was deduced from a standard curve. A student t-test was performed to determine significant differences, which are indicated as asterisk $(* * *, P<0.001)$. Error bars represent the standard deviation of three biological repeats. (C) Heterologous expression and secretion of FvRibo1 in $\boldsymbol{U}$. hordei strain DS200 in planta. Ustilago hordei strain DS200 and DS200 expressing FvRibol (encoding a secreted ribotoxin) of Fusarium verticillioides with UHOR_02700 signal peptide and under the control of the UHOR_02700 promoter (for only in planta expression) were inoculated on susceptible 12-dayold barley seedlings. Macroscopic pictures were taken at $6 \mathrm{dpi}$. Autofluorescence pictures were taken to see better cell death by using Gel-Doc (Bio-Rad). (D) WGA-AF488/Propidium iodide staining was performed to visualize the colonization of DS200-FvRibo1 in barley leaves compared to DS200. While green signal indicates fungal colonization, the red signal represents the plant cell walls.

\section{Discussion}

Plant pathogenic smut fungi are non-obligate biotrophic fungal pathogens, which can infect many economically important crops, such as maize, wheat, barley, oat and sugar cane. Recent comparative genome analysis of five plant pathogenic smut fungi, including $U$. hordei, $U$. maydis, Sporisorium reilianum, Sporisorium scitamineum and Melanopsichum pennsylvanicum, showed that all of these smut fungi have relatively small genomes (about 20 Mbp) [43]. These genomic features make smut fungi excellent candidates for functional genetic and genomic approaches. The availability of complete genome assemblies, available transcriptomics data and the possibility of performing reverse genetics make the $U$. hordeibarley system a potential model for studying the molecular basis of plant-pathogen interactions. To enable and speed-up functional genetics in the $U$. hordei-barley interaction, we have established several molecular tools, including a solopathogenic $U$. hordei strain, heterologous gene expression and an efficient CRISPR/Cas9 gene editing system.

\section{Establishment of a solopathogenic strain}

The generation of haploid solopathogenic $U$. hordei strains, which express an active $\mathrm{bE} / \mathrm{bW}$ heterodimer and form infectious filaments without having to fuse with a mating partner, increases the efficiency of genetic transformation by reducing lab workload, since no duplicate mutants in opposite mating partners needed. Quantification of appressoria formation and penetration efficiency of solopathogenic strains showed that there is no significant difference compared to wild-type strain on barley leaves. This result indicates that the solopathogenic strains can be used for functional characterization of virulence factors during barley leaf colonization. Although the barley leaf infection assay did not show any difference in colonization of solopathogenic and wild-type strains, in barley seed infection assays, the solopathogenic strains rarely colonized barley inflorescences and produced teliospores compared to the wild-type strain. This observation indicates that the solopathogenic strain is 
454 only weakly pathogenic in systemic colonization of barley and may not produce all virulence

455 factors/effectors needed at the later stages of infection. Accordingly, two generated 456 solopathogenic U. maydis strains, SG200 and CL13, also showed attenuated virulence 457 compared to wild-type strains [44, 45]. Moreover, CL13, which is generated by replacement 458 of only compatible $b$ loci, shows a more attenuated virulence compared to the SG200 strain, 459 which is generated by replacement of both compatible $a$ and $b$ loci [44, 45]. In our 460 experiments, the $U$. hordei solopathogenic strains DS199 (with only compatible $b$ alleles) and 461 DS200 (with both compatible $a$ and $b$ alleles) showed similar rates of virulence during barley 462 penetration. This suggests that the presence of a compatible $a$ loci is more important for the 463 formation of infectious filaments in $U$. maydis, which has a tetrapolar mating system, than in $464 U$. hordei, which has a bipolar mating system [19].

465 Recently, Schuster et al., (2016) established a CRISPR/Cas9 gene editing system for $U$. 466 maydis with $~ 70 \%$ efficiency [46]. By using a similar approach, we achieved a very efficient 467 CRISPR/Cas9-HF based system in $U$. hordei with $\sim 83 \%$ gene editing efficiency in progeny. 468 CRISPR/Cas9 gene editing of the U. hordei Flyl gene, a fungalysin metalloprotease involved 469 in fungal cell separation [41], resulted in an impaired cell separation phenotype in DS200, 470 indicating the functional conservation of this protein among smut fungi. Thus, establishment 471 of both solopathogenic strains and a CRISPR/Cas9 gene editing system allow fast and 472 efficient reverse genetic approaches in $U$. hordei.

Ultrastructural analysis of the Ustilago hordei-barley biotrophic interphase

475 During barley leaf colonization, U. hordei enters in the host cell without breaching the host plasma membrane. Thus, the $U$. hordei-barley interaction is mediated through the biotrophic interphase, which comprises the fungal cell wall (FCW), electron-dense and electrontranslucent extracellular matrixes (edECM and etECM) and the plant cell wall (PCW) (in some part of colonized tissues). Presence of vesicles in the fungal cytoplasm close to the hyphal tip and in the surrounding plant cytoplasm as well as of plant multivesicular bodies close to fungal penetration sites indicates that the biotrophic interphase is very active site. Some vesicles appeared to be in the process of either fusing with or pinching off the plant cell membrane. The edECM of unknown composition surrounds the FCW and in some regions, its outer surface shows irregular patterns with small protrusions. At contact sites with the PCW and at cell-to-cell penetration sites, $U$. hordei accumulates a thicker edECM and it seems that the material causing the electron-density of the ECM diffuses into the adjacent PCW. This observation indicates that the interphase between the plant cell membrane and the edECM is 
quite active. The plant apoplastic space contains a wide range of defense components, such as glycoside hydrolyses, proteases, peroxidases, antimicrobial proteins and secondary metabolites, which collectively contribute to plant immunity [47]. U. hordei hyphae may secrete the edECM to prevent access of these plant-derived defense components to the fungal cell. Since the thickness of edECM gets higher with contact to the host PCW, one can also hypothesize that it is required for anchoring to the PCW to increase cell-to-cell penetration efficiency. Similar edECM were also observed for other smut fungi, such as the maize smut fugus U. maydis and Ustacystis waldsteiniae (on Waldsteinia geoides host), rust as well as powdery mildew (Hyaloperonospora parasitica) during host colonization [48-51]. Although the content of edECM is unknown, immunogold gold labeling with (1-3)- $\beta$-glucan specific antibodies revealed presence of callose in the electron-translucent extracellular matrix (etECM). In incompatible host-pathogen interactions, callose deposition at the site of infection is a hallmark of plant immune response; however, in compatible interactions, successful pathogens (like $U$. hordei) can suppress the host defense response, including callose deposition [52]. Therefore, the presence of callose in the etECM indicates that $U$. hordei may have an ability to use callose at the biotrophic interphase as an additional carbon source. Up-regulation of several 1,3 beta-glucanase encoding genes in $U$. hordei during host colonization might be involved in this process [53].

To reach the host vascular bundles, $U$. hordei grows/moves mostly intracellularly from cellto-cell. Detailed observation revealed that at the site of cell-to-cell penetration, the $U$. hordei hypha developed a swollen structure, which resembled an appressorium. A similar phenotype of swollen hyphal tips was also observed in U. maydis during cell-to-cell penetration in maize [54]. Formation of this structure may increase the penetration efficiency of smut fungi from cell-to-cell movements. In Ökmen et al., (2018), we have reported that $U$. hordei intracellular hyphae develop lobed haustoria-like structures during barley colonization [20]. One infected host cell could have more than one haustorium. Haustorial structures of $U$. hordei were distinguished from the normal hyphae by their bigger size and inter-connected lobular shapes. While both rust and powdery mildew haustoria are formed from extracellular fungal hyphae $[55,56], U$. hordei haustoria structures originate from intracellular hyphae. In addition, a structure comparable to the neckband of rust haustoria that separates the haustorial matrix from the apoplast was not seen in our analysis [57]. Detailed transmission electron micrographs of $U$. hordei haustoria also showed that these structures contain large vacuoles with a fine-granular content and intraluminal vesicles. 


\section{Heterologous expression of fungal effectors in Ustilago hordei}

Both smuts and rusts are plant pathogenic fungi belonging to the division Basidiomycotina

[58]. In addition to their phylogenetic relationships, smuts and rusts (obligate biotroph) have similar biotrophic life styles, in which they require an intimate association with their hosts to acquire nutrients and complete their pathogenic lifecycles. Apart from their phylogenetic relationships and similar lifestyles, U. hordei, Bgh and Pgt also form comparable intracellular haustorial structures, secrete edECM, and infect the same host plant species. Due to hostspecific adaptations during co-evolution, entirely different pathogens that share the same host can independently develop different types of effectors that interact with the same target. Accordingly, Avr2 of C. fulvum (fungus) [59-61], EPIC1 and EPIC2B of Phytophthora infestans (oomycete) [62], Gr-VAP1 of Globodera rostochiensis (nematode) [63] and Cip1 from Pseudomonas syringae (bacterium) [64] can interact and inhibit tomato cysteine protease, Rcr3. Therefore, the $U$. hordei-barley pathosystem allows to perform reverse genetics for Bgh and Pgt effectors in the same host system and subsequently to identify their virulence functions.

Successful heterologous expression and secretion of the GUS-mCherry recombinant protein in $U$. hordei during barley colonization demonstrates that the designed concept is feasible. Some of the GUS-mCherry was also cleaved in apoplastic space of barley leaf, indicating Cterminal processing of this protein. For further proof of concept that $U$. hordei can express and secrete functional effectors from different fungi, a very robust Avr4/Cf4 pair was used to induce HR-mediated cell death. To this end, Avr4 avirulence gene from C. fulvum was expressed in DS200 in vitro. Induction of Cf4-mediated HR only in the presence of culture filtrate of DS200-Avr4 strain indicates that DS200-Avr4 expresses and secretes functional Avr4 from a biotrophic $C$. fulvum that can be recognized by $\mathrm{Cf} 4$ resistance protein. In a similar way, in planta expression and secretion of a plant cytotoxic Ribol (FvRibo1) from $F$. verticillioides in DS200 was confirmed by using only in planta expressed $U$. hordei promoter. Heterologous expression of the FvRibo1 in DS200 negatively affects the colonization of this biotrophic smut fungus in barley. The WGA-AF488/PI staining and biomass quantification assays display that DS200-FvRibo1 hardly moves and colonizes vascular bundles and showed significantly reduced fungal biomass compared to DS200 strain. Moreover, macroscopic and microscopic observations of focal necrotic spots on barley leaves indicate that FvRibo1 is also 
556 plant pathogenic fungi showed that the solopathogenic U. hordei DS200 strain can be used for

557 functional characterization of effectors from biotrophic phytopathogens as well.

558 Biotrophic infection of barley leaves, formation of intracellular hyphae and haustorial 559 structures as well as the molecular tools presented in this study make the $U$. hordei 560 pathosystem as a useful platform for the functional analysis of effector proteins from 561 biotrophic fungi.

562

563 Supplementary Material

564 Figure S1 Ustilago hordei-barley infection assay.

565 Figure S2 Cell-to-cell penetration of Ustilago hordei.

566 Figure S3 Ultrastructural features of the Ustilago hordei during barley colonization.

567 Table S1. Plasmids and primers used in this study.

\section{Acknowledgements}

570 We thank Ila Rouhara for technical assistance for TEM. We acknowledge Regine Kahmann and the Max-Planck-Institute for Terrestrial Microbiology, Marburg, Germany, for providing generous support and access to infrastructure. This research is supported by the European Research Council under the European Union's Horizon 2020 research and innovation program (consolidator grant conVIRgens, ID 771035).

\section{Author contributions}

577 BÖ and GD designed the research; BÖ, UN and DS performed experimental work, BÖ 578 analyzed the data. GB provided materials and advice on the experimental design, BÖ wrote 579 the paper with input from GD, UN and GB.

580

581 Conflicts of Interest

582 The authors have no conflicts of interest

\section{References}

585 1. Spanu, P. D.; Abbott, J. C.; Amselem, J.; Burgis, T. A.; Soanes, D. M.; Stueber, K.; van 586 Themaat, E. V. L.; et al. Genome expansion and gene loss in powdery mildew fungi reveal 587 tradeoffs in extreme parasitism. Science 2010, 330 (6010), 1543-1546. 
588

589

590

591

592

593

594

595

596

597

598

599

600

601

602

603

604

605

606

607

608

609

610

611

612

613

614

615

616

617

618

619

620

621

2. Hacquard, S.; Joly, D. L.; Lin, Y.-C.; Tisserant, E.; Feau, N.; Delaruelle, C.; Legue, V.; Kohler, A.; et al. A comprehensive analysis of genes encoding small secreted proteins identifies candidate effectors in Melampsora larici-populina (poplar leaf rust). Molecular Plant-Microbe Interactions 2012, 25 (3), 279-293.

3. Pedersen, C.; van Themaat, E. V. L.; McGuffin, L. J.; Abbott, J. C.; Burgis, T. A.; Barton, G.; et al. Structure and evolution of barley powdery mildew effector candidates. BMC Genomics 2012, 13, 694.

4. Duplessis, S.; Cuomo, C. A.; Lin, Y.-C.; Aerts, A.; Tisserant, E.; Veneault-Fourrey, C.; Joly, D. L.; et al. Obligate biotrophy features unraveled by the genomic analysis of rust fungi. Proceedings of the National Academy of Sciences of the United States of America 2011, 108 (22), 9166-9171.

5. O'Connell, R. J.; Thon, M. R.; Hacquard, S.; Amyotte, S. G.; Kleemann, J.; Torres, M. F.; Damm, U.; Buiate, E. A.; et al. Lifestyle transitions in plant pathogenic Colletotrichum fungi deciphered by genome and transcriptome analyses. Nature Genetic 2012, 44 (9), 1060-5.

6. de Wit, P. J.; van der Burgt, A.; Okmen, B.; Stergiopoulos, I.; Abd-Elsalam, K. A.; Aerts, A. L.; Bahkali, A. H.; et al. The genomes of the fungal plant pathogens Cladosporium fulvum and Dothistroma septosporum reveal adaptation to different hosts and lifestyles but also signatures of common ancestry. PLoS Genetics 2012, 8 (11), e1003088.

7. Zhao, Z.; Liu, H.; Wang, C.; Xu, J.-R. Comparative analysis of fungal genomes reveals different plant cell wall degrading capacity in fungi. BMC Genomics 2013, 14 (1), 274.

8. Rodriguez-Moreno, L.; Ebert, M. K.; Bolton, M. D.; Thomma, B. P. H. J. Tools of the crook- infection strategies of fungal plant pathogens. The Plant Journal 2018, 93 (4), 664674.

9. Germain, H.; Joly, D. L.; Mireault, C.; Plourde, M. B.; Letanneur, C.; Stewart, D.; Morency, M.-J.; Petre, B.; Duplessis, S.; Séguin, A. Infection assays in Arabidopsis reveal candidate effectors from the poplar rust fungus that promote susceptibility to bacteria and oomycete pathogens. Molecular plant pathology 2018, 19 (1), 191-200.

10. Xu, Q.; Tang, C.; Wang, L.; Zhao, C.; Kang, Z.; Wang, X. Haustoria - arsenals during the interaction between wheat and Puccinia striiformis f. sp. tritici. Molecular plant pathology 2020, 21 (1), 83-94.

11. Fabro, G.; Steinbrenner, J.; Coates, M.; Ishaque, N.; Baxter, L.; Studholme, D. J.; Koerner, E.; Allen, R. L.; et al. Multiple candidate effectors from the oomycete pathogen Hyaloperonospora arabidopsidis suppress host plant immunity. PLoS Pathogens 2011, 7 (11). 
622 12. Sohn, K. H.; Lei, R.; Nemri, A.; Jones, J. D. G. The downy mildew effector proteins 623 ATR1 and ATR13 promote disease susceptibility in Arabidopsis thaliana. Plant Cell 2007, 19 624 (12), 4077-4090.

625 13. Upadhyaya, N. M.; Ellis, J. G.; Dodds, P. N. A bacterial type III secretion-based delivery 626 system for functional assays of fungal effectors in cereals. Methods Mol Biol 2014, 1127, $627 \quad 277-90$.

628 14. Montenegro Alonso, A. P.; Ali, S.; Song, X.; Linning, R.; Bakkeren, G. UhAVR1, an HR629 triggering avirulence effector of Ustilago hordei, is secreted via the ER-Golgi pathway, 630 localizes to the cytosol of barley cells during in planta-expression, and contributes to 631 virulence early in infection. J Fungi 2020, 6 (3).

632 15. Ramachandran, S. R.; Yin, C.; Kud, J.; Tanaka, K.; Mahoney, A. K.; Xiao, F.; Hulbert, S. 633 H. Effectors from wheat rust fungi suppress multiple plant defense responses. Phytopathology 634 2017, $107(1), 75-83$.

635 16. Panwar, V.; McCallum, B.; Bakkeren, G. Host-induced gene silencing of wheat leaf rust 636 fungus Puccinia triticina pathogenicity genes mediated by the Barley stripe mosaic virus. 637 Plant Mol Biol 2013, 81 (6), 595-608.

638 17. Yin, C.; Hulbert, S. H. Host-induced gene silencing (higs) for elucidating Puccinia gene 639 function in wheat. Methods Mol Biol 2018, 1848, 139-150.

640 18. Yang, Q.; Huai, B.; Lu, Y.; Cai, K.; Guo, J.; Zhu, X.; Kang, Z.; Guo, J. A stripe rust 641 effector Pst18363 targets and stabilises TaNUDX23 that promotes stripe rust disease. New 642 Phytologist 2020, 225 (2), 880-895.

643 19. Zuo, W.; Okmen, B.; Depotter, J. R. L.; Ebert, M. K.; Redkar, A.; Villamil, J. M.; 644 Doehlemann, G. Molecular interactions between smut fungi and their host plants. Annual 645 Review of Phytopathology 2019, 57, 411-430.

646 20. Ökmen, B.; Mathow, D.; Hof, A.; Lahrmann, U.; Aßmann, D.; Doehlemann, G. Mining 647 the effector repertoire of the biotrophic fungal pathogen Ustilago hordei during host and non648 host infection. Molecular plant pathology 2018, 12, 2603-2622.

649 21. Hu, G. G.; Linning, R.; Bakkeren, G. Sporidial mating and infection process of the smut 650 fungus, Ustilago hordei, in susceptible barley. Canadian Journal of Botany 2002, 80 (10), $651 \quad 1103-1114$.

652 22. Chong, J.; Harder, D.; Rohringer, R. Cytochemical studies on Puccinia graminis f. sp. 653 tritici in a compatible wheat host. II. Haustorium mother cell walls at the host cell penetration 654 site, haustorial walls, and the extrahaustorial matrix. Canadian Journal of Botany 2011, 64, $655 \quad 2561-2575$. 
656 23. Hippe-Sanwald, S.; Hermanns, M.; Somerville, S. C. Ultrastructural comparison of 657 incompatible and compatible interactions in the barley powdery mildew disease. Protoplasma 1992, $168(1), 27-40$.

24. Sambrook, J.; Fritsch, E. F.; Maniatis, T. Molecular cloning: a laboratory manual. Cold Spring Harbor Laboratory, Cold Spring Harbor, New York. 1989. maydis. Fungal Biology 2020, 124 (3), 228-234. Nature Methods 2014, 11 (2), 122-3.

665 27. Gibson, D. G.; Young, L.; Chuang, R.-Y.; Venter, J. C.; Hutchison, C. A.; Smith, H. O. 666 Enzymatic assembly of DNA molecules up to several hundred kilobases. Nature Methods 2009, 6 (5), 343-345.

28. Kämper, J. A PCR-based system for highly efficient generation of gene replacement mutants in Ustilago maydis. Molecular Genetics and Genomics 2004, 271 (1), 103-110. 29. van der Linde, K.; Hemetsberger, C.; Kastner, C.; Kaschani, F.; van der Hoorn, R. A.;

671 Kumlehn, J.; Doehlemann, G. A maize cystatin suppresses host immunity by inhibiting 672 apoplastic cysteine proteases. The Plant Cell 2012, 24 (3), 1285-300.

673 30. Mueller, A. N.; Ziemann, S.; Treitschke, S.; Assmann, D.; Doehlemann, G. Compatibility 674 in the Ustilago maydis-maize interaction requires inhibition of host cysteine proteases by the fungal effector Pit2. PLoS Pathogens 2013, 9 (2), e1003177. 31. Wawra, S.; Fesel, P.; Widmer, H.; Neumann, U.; Lahrmann, U.; Becker, S.; Hehemann, J.-H.; Langen, G.; Zuccaro, A. FGB1 and WSC3 are in planta-induced $\beta$-glucan-binding fungal lectins with different functions. New Phytologist 2019, 222 (3), 1493-1506. 32. McDonald, K. L. Out with the old and in with the new: rapid specimen preparation procedures for electron microscopy of sectioned biological material. Protoplasma 2014, 251 681 (2), 429-448.

682 33. Micali, C. O.; Neumann, U.; Grunewald, D.; Panstruga, R.; O'Connell, R. Biogenesis of a 683 specialized plant-fungal interface during host cell internalization of Golovinomyces orontii haustoria. Cellular Microbiology 2011, 13 (2), 210-26. Hayat M A (ed), New York, Academic Press: 1987; 1-22. 
690

691

692

693

694

695

696

697

698

699

700

701

702

703

704

705

706

707

708

709

710

711

712

713

714

715

716

717

718

719

720

721

722

723

sections prepared by high pressure freezing/freeze substitution. Journal of Microscopy 2001, $201(1), 77-83$.

36. Reynolds, E. S. The use of lead citrate at high $\mathrm{pH}$ as an electron-opaque stain in electron microscopy. Journal of Cell Biology 1963, 17 (1), 208-12.

37. Banuett, F. Genetics of Ustilago maydis, a fungal pathogen that induces tumors in maize. Annual Review of Genetics 1995, 29, 179-208.

38. Kahmann, R.; Steinberg, G.; Basse, C.; Feldbrügge, M.; Kämper, J. Ustilago maydis, the causative agent of corn smut disease. In: Fungal Pathology, Kronstad, J. W., Ed. Kluwer academic publishers: Dodrecht, the Netherlands, 2000; 347-371.

39. Khrunyk, Y.; Münch, K.; Schipper, K.; Lupas, A. N.; Kahmann, R., The use of FLPmediated recombination for the functional analysis of an effector gene family in the biotrophic smut fungus Ustilago maydis. New Phytologist 2010, 187 (4), 957-68.

40. Mendoza-Mendoza, A.; Berndt, P.; Djamei, A.; Weise, C.; Linne, U.; Marahiel, M.; Vraneš, M.; Kämper, J.; Kahmann, R. Physical-chemical plant-derived signals induce differentiation in Ustilago maydis. Molecular Microbiology 2009, 71 (4), 895-911.

41. Ökmen, B.; Kemmerich, B.; Hilbig, D.; Wemhoner, R.; Aschenbroich, J.; Perrar, A.; Huesgen, P. F.; Schipper, K.; Doehlemann, G. Dual function of a secreted fungalysin metalloprotease in Ustilago maydis. New Phytologist 2018, 220 (1), 249-261.

42. Thomas, C. M.; Jones, D. A.; Parniske, M.; Harrison, K.; Balint-Kurti, P. J.; Hatzixanthis, K.; Jones, J. D. Characterization of the tomato Cf-4 gene for resistance to Cladosporium fulvum identifies sequences that determine recognitional specificity in Cf-4 and Cf-9. The Plant Cell 1997, 9 (12), 2209-2224.

43. Schuster, M.; Schweizer, G.; Kahmann, R. Comparative analyses of secreted proteins in plant pathogenic smut fungi and related basidiomycetes. Fungal Genetics and Biology 2018, 112, 21-30.

44. Bölker, M.; Genin, S.; Lehmler, C.; Kahmann, R., Genetic regulation of mating and dimorphism in Ustilago maydis. Canadian Journal of Botany 1995, 73 (S1), 320-325.

45. Djamei, A.; Schipper, K.; Rabe, F.; Ghosh, A.; Vincon, V.; Kahnt, J.; Osorio, S.; Tohge, T.; Fernie, A. R.; Feussner, I.; et al. Metabolic priming by a secreted fungal effector. Nature 2011, 478, 395-398.

46. Schuster, M.; Schweizer, G.; Reissmann, S.; Kahmann, R. Genome editing in Ustilago maydis using the CRISPR-Cas system. Fungal Genetics and Biology 2016, 89, 3-9.

47. Hoson, T. Apoplast as the site of response to environmental signals. Journal of Plant Research 1998, 111 (1101), 167-77. 
48. Snetselaar, K. M.; Mims, C. W. Light and electron microscopy of Ustilago maydis hyphae in maize. Mycol Research 1994, 98 (3), 347-355.

49. Bauer, R.; Oberwinkler, F.; Mendgen, K. Cellular interaction of the smut fungus Ustacystis waldsteiniae. Canadian Journal of Botany 1995, 73 (6), 867-883.

50. Mims, C. W.; Richardson, E. A.; Holt Iii, B. F.; Dangl, J. L. Ultrastructure of the hostpathogen interface in Arabidopsis thaliana leaves infected by the downy mildew Hyaloperonospora parasitica. Canadian Journal of Botany 2004, 82 (7), 1001-1008.

51. Mims, C. W.; Rodriguez-Lother, C.; Richardson, E. A. Ultrastructure of the hostpathogen interface in daylily leaves infected by the rust fungus Puccinia hemerocallidis. Protoplasma 2002, 219 (3), 221-226.

52. Gaudet, D. A.; Wang, Y.; Penniket, C.; Lu, Z. X.; Bakkeren, G.; Laroche, A. Morphological and molecular analyses of host and nonhost interactions involving barley and wheat and the covered smut pathogen Ustilago hordei. Molecular Plant-Microbe Interactions 2010, 23 (12), 1619-34.

53. Ökmen, B.; Mathow, D.; Hof, A.; Lahrmann, U.; Aßmann, D.; Doehlemann, G. Mining the effector repertoire of the biotrophic fungal pathogen Ustilago hordei during host and nonhost infection. Molecular Plant Pathology 2018, 19 (12), 2603-2622.

54. Doehlemann, G.; Wahl, R.; Horst, R. J.; Voll, L. M.; Usadel, B.; Poree, F.; Stitt, M.; PonsKühnemann, J.; Sonnewald, U.; Kahmann, R.; Kämper, J. Reprogramming a maize plant: transcriptional and metabolic changes induced by the fungal biotroph Ustilago maydis. Plant Journal 2008, 56 (2), 181-195.

55. Szabo, L. J.; Bushnell, W. R. Hidden robbers: the role of fungal haustoria in parasitism of plants. Proceedings of the National Academy of Sciences of the United States of America 2001, 98 (14), 7654-7655.

56. Voegele, R. T.; Mendgen, K. Rust haustoria: nutrient uptake and beyond. New Phytologist 2003, 159 (1), 93-100.

57. Mims, C. W. Using electron microscopy to study plant pathogenic fungi. Mycologia 1991, $83(1), 1-19$.

58. Spatafora, J. W.; Aime, M. C.; Grigoriev, I. V.; Martin, F.; Stajich, J. E.; Blackwell, M. The fungal tree of life: from molecular systematics to genome-scale phylogenies. Microbiology spectrum 2017, 5 (5).

59. Kruger, J.; Thomas, C. M.; Golstein, C.; Dixon, M. S.; Smoker, M.; Tang, S. K.; Mulder, L.; Jones, J. D. G. A tomato cysteine protease required for Cf-2-dependent disease resistance and suppression of autonecrosis. Science 2002, 296 (5568), 744-747. 
758

759

760

761

762

763

764

765

766

767

768

769

770

771

772

773

774

775

776

777

778

779

60. Rooney, H. C. E.; van 't Klooster, J. W.; van der Hoorn, R. A. L.; Joosten, M. H. A. J.; Jones, J. D. G.; de Wit, P. J. G. M. Cladosporium Avr2 inhibits tomato Rcr3 protease required for Cf-2-dependent disease resistance. Science 2005, 308 (5729), 1783-1786.

61. van Esse, H. P.; van't Klooster, J. W.; Bolton, M. D.; Yadeta, K. A.; van Baarlen, P.; Boeren, S.; Vervoort, J.; de Wit, P. J. G. M.; Thomma, B. P. H. J. The Cladosporium fulvum virulence protein Avr2 inhibits host proteases required for basal defense. Plant Cell 2008, 20 (7), 1948-1963.

62. Song, J.; Win, J.; Tian, M.; Schornack, S.; Kaschani, F.; Ilyas, M.; van der Hoorn, R. A. L.; Kamoun, S. Apoplastic effectors secreted by two unrelated eukaryotic plant pathogens target the tomato defense protease Rcr3. Proceedings of the National Academy of Sciences of the United States of America 2009, 106 (5), 1654-1659.

63. Lozano-Torres, J. L.; Wilbers, R. H. P.; Gawronski, P.; Boshoven, J. C.; FinkersTomczak, A.; Cordewener, J. H. G.; et al. Dual disease resistance mediated by the immune receptor $\mathrm{Cf}-2$ in tomato requires a common virulence target of a fungus and a nematode. Proceedings of the National Academy of Sciences of the United States of America 2012, 109 (25), 10119-10124.

64. Shindo, T.; Kaschani, F.; Yang, F.; Kovács, J.; Tian, F.; Kourelis, J.; Hong, T. N.; Colby, T.; Shabab, M.; Chawla, R.; et al. Screen of non-annotated small secreted proteins of Pseudomonas syringae reveals a virulence factor that inhibits tomato immune proteases. PLoS Pathogens 2016, 12 (9), e1005874. 
Figure S1 Ustilago hordei-barley infection assay. Dehulled and surface sterilized barley Golden Promise seeds were inoculated with the $U$. hordei wild-type and DS200 strains. Approximately 3-4 months after inoculation, disease symptoms were observed at barley heading.

Figure S2 (A-D) Cell-to-cell penetration of Ustilago hordei. U. hordei primarily grows intracellularly at 8 dpi in barley leaves. The fungal hyphae grow through the plant cells and frequently branch. The $U$. hordei hyphal cell wall was surrounded by an extracellular matrix of electron-dense (edECM) (between 50-500 nm thickness) of unknown composition. The extracellular matrix gets thicker where the hypha was in contact with the plant cell wall (white arrowheads in A). edECM: electron-dense extracellular matrix; LB: Lipid bodies; PCW: Plant Cell Wall.

Figure S3 (A-C) Ultrastructural features of the Ustilago hordei during barley colonization. The $U$. hordei electron-dense extracellular matrix (edECM) gets thicker where the hypha was in contact with the plant cell wall (PCW) and has a highly irregular surface structure (C). (D-F) U. hordei hyphae contain free ribosomes, strands of endoplasmic reticulum (ER), mitochondria (m), nuclei (n), as well as lipid bodies (LB), vesicles (Ve) and vacuoles (Va). Transmission electron micrographs also showed the presence of closely paired nuclei, tightly associated with mitochondria (F). Vesicles (Ve) with cores of different electron densities and multivesicular bodies (MVB) were detected in hyphal tips and in the plant cytoplasm close to fungal penetration sites, respectively $(\mathbf{B}, \mathbf{C}, \mathbf{F})$.

Table S1. Plasmids and primers used in this study. 
807

808

809

810

811

812

813

814

815

816

817

818

819

820

821

822

823

824

825

826

827

828

829

830

831

832

833

834

835

836

837

838

839

840

\section{Figure Legends}

Figure 1 Generation of a solopathogenic Ustilago hordei strain (A) Filamentation test on charcoal plate. U. hordei wild-type strains 4857-4 MAT-1, 4857-5 MAT-2, mating of 4857-4 MAT-1X4857-5 MAT-2, solopathogenic DS199 and DS200 strains. Pictures were taken after 3 days incubation at RT. (B) Appressoria formation ability of Ustilago hordei strains on parafilm. Mating of $U$. hordei wild-type 4857-4 MAT-1 and 4857-5 MAT-2, solopathogenic DS199, solopathogenic DS200. Yellow arrowheads indicate appressoria. Pictures were taken after 24 hours incubation (C) Disease development of different Ustilago hordei strains on barley. Mating of $U$. hordei wild-type 4857-4 MAT-1 and 4857-5 MAT-2 at 3 dpi, solopathogenic DS199 at $3 \mathrm{dpi}$, solopathogenic DS200 at 3 days post inoculation (dpi). Following WGA-AF488/Propidium iodide staining, fungal cell walls are shown in green and plant cell walls in red. (D) Quantification of appressoria formation for $\boldsymbol{U}$. hordei wild-type 4857-4 MAT1, solopathogenic DS199 and DS200 on plant. (E) Quantification of penetration efficiency for $\boldsymbol{U}$. hordei wild-type 4857-4 MAT1, solopathogenic DS199 and DS200 on plant.

Figure 2 (A-H) Transmission electron microscopy micrographs of wild-type Ustilago hordei infected barley leaves. (A, B) Biotrophic interphase in the $U$. hordei-barley interaction. During host colonization, U. hordei invaginates the host cell membrane without breaching it. The host-pathogen interaction mainly takes place within this biotrophic interphase (BIP), which consists of fungal cell wall (FCW), electron-dense extracellular matrix (edECM) and electron-translucent extracellular matrix (etECM). (C, D) Formation of vesicles at the hyphal tip of $\boldsymbol{U}$. hordei. Fungal vesicles (Ve) with cores of different electron density and plant multivesicular bodies (MVB) were detected at hyphal tips and in the plant cytoplasm close to fungal penetration sites, respectively. (E, F) Immunogold labeling of callose with a monoclonal antibody recognizing (1-3)- $\beta$-glucan epitopes. Callose accumulation was detected at the electron-translucent ECM (IdECM) site (yellow arrowheads). (G, H) Cell-to-cell penetration of $\boldsymbol{U}$. hordei. U. hordei primarily grows intracellularly at $8 \mathrm{dpi}$ in barley leaves. When the fungal hyphae penetrate a new plant cell, the hypha gets thickened at the site of cell-to-cell passage, resembling appressorial structures (G). The edECM gets thicker where the hypha was in contact with the plant cell wall (yellow arrowheads) (H) and electron-dense material can also diffuse into adjacent part of the plant cell wall (red arrowhead) (H). (I-M) Haustoria formation during host colonization. $U$. hordei grows intracellularly and forms haustorial structures in barley cells. (I, J) WGA- 
841 AF488/Propidium iodide staining was performed to visualize $U$. hordei at 8 dpi under 842 confocal/ fluorescent microscopy. (K-M) Transmission electron micrographs showing 843 different planes of section through haustoria. Haustorial structures were distinguished from 844 hyphae by their bigger size and inter-connected lobular shapes. Yellow arrowheads (L) point 845 out the connections between haustorial lobes. U. hordei haustoria possess large vacuoles with 846 a granular lumen containing vesicles of different size (M, yellow arrowheads; magnification 847 of inset in L). BIP: Biotrophic Interphase; FCW: Fungal Cell Wall; FPM: Fungal Plasma 848 Membrane; H: Hypha; Ha: Haustorium; edECM: electron-dense extracellular matrix; etECM: 849 electron-translucent extracellular matrix; LB: Lipid bodies; MVB: Multi Vesicular Body; 850 PCW: Plant Cell Wall; PPM: Plant Plasma Membrane; Ve: Vesicles.

851

Fig 3. (A-B) Heterologous expression of GusA-mCherry in Ustilago hordei. (A) GusAmCherry was heterologously expressed in solopathogenic strain DS200 under control of the UHOR_02700 promotor with or without signal peptide for extracellular secretion. \pm SP-GusAmCherry DS200 strains were inoculated on barley seedlings and at 4 dpi confocal microscopy was performed to monitor expression and localization of recombinant proteins. While + SPGusA-mCherry is secreted around the tip of the invasive hyphae, -sp-GusA-mCherry localizes in the fungal cytoplasm. The white graphs indicate the mCherry signal intensity along the diameter of the hyphae (illustrated by white lines in the image). (B) Western blot analysis was performed with apoplastic fluid isolated from barley leaves infected with \pm SP-GusA-mCherry DS200 strains. While a band corresponding to secreted +SP-GusA-mCherry (at 100 kDa) and free mCherry (at $27 \mathrm{kDa}$ ) in isolated apoplastic fluid was detected, no band corresponding to cytoplasmic -sp-GusA-mCherry could be detected. Anti-RFP antibody was used for western blot. (C-D) Establishment of CRISPR/Cas9 gene editing system for Ustilago hordei. (C) Codon optimized Cas 9 was cloned into p123 plasmid under the control of Hsp70 promoter. The $U$. hordei pU6 promotor was used to express sgRNA for the targeted gene.

867 Carboxin resistance was used as selection marker. (D) U. hordei Flyl gene, a fungalysin 868 metalloprotease involved in fungal cell separation, was edited via CRISPR/Cas9 system for knock-out. While DS200 sporidia showed normal growth, DS2004uhfly1 cells were impaired in cell separation.

871 Figure 4 Heterologous expression of fungal effectors in Ustilago hordei. (A) 872 Heterologous expression and secretion of CfAvr4 in $U$. hordei DS200 strain in vitro. $U$. hordei strain DS200 expressing CfAvr4 of Cladosporium fulvum with UHOR_02700 signal 
875 of Fusarium verticillioides with UHOR_02700 signal peptide and under the control of

876 pUHOR_02700 promoter (for expression in planta only) were grown in $\mathrm{YEPS}_{\text {light }}$ liquid

877 medium till OD:1.0. The U. hordei cell suspensions were centrifuged and the culture filtrates

878 (CF) of each sample were infiltrated into tobacco leaves expressing Cf4 resistant protein that 879 can recognize CfAvr4 and induce cell death by means of hypersensitive response. The culture 880 filtrates from $U$. hordei DS200 and DS200-FvRibo1 strains were used as negative controls.

881 Pictures were taken at $5 \mathrm{dpi}$. Autofluorescence of infected leaves was imaged to more easily 882 see sites of cell death by using Gel-Doc (Bio-Rad). (B) Biomass quantification of DS200-

883 FvRibo1 in barley leaves. The virulence of the $U$. hordei DS200 and two independent 884 DS200-FvRibo1 strains was assessed by fungal biomass quantification from DNA isolated 885 from infected barley leaves at $6 \mathrm{dpi}$. The Ppil gene of $U$. hordei was used as a standard for 886 qPCR. The fungal biomass was deduced from a standard curve. A student t-test was 887 performed to determine significant differences, which are indicated as asterisk (***, $P<$ 888 0.001). Error bars represent the standard deviation of three biological repeats. (C) 889 Heterologous expression and secretion of FvRibo1 in U. hordei strain DS200 in planta. 890 Ustilago hordei strain DS200 and DS200 expressing FvRibol (encoding a secreted ribotoxin) 891 of Fusarium verticillioides with UHOR_02700 signal peptide and under the control of the 892 UHOR_02700 promoter (for only in planta expression) were inoculated on susceptible 12893 day-old barley seedlings. Macroscopic pictures were taken at 6 dpi. Autofluorescence pictures 894 were taken to see better cell death by using Gel-Doc (Bio-Rad). (D) WGA895 AF488/Propidium iodide staining was performed to visualize the colonization of DS200896 FvRibo1 in barley leaves compared to DS200. While green signal indicates fungal 897 colonization, the red signal represents the plant cell walls. 